\title{
Experience with implanted pacemakers: technical considerations
}

\author{
J. G. DAVIES AND HAROLD SIDDONS \\ From St. George's Hospital, London
}

Between April 1960 and September 1964, 79 patients were treated with implanted pacemakers. We record here the technical difficulties encountered and the steps taken to overcome them. In all cases the indication for artificial pacing was failure of medical treatment to prevent Stokes-Adams attacks or to relieve cardiac failure due to a slow pulse. Most, but not all, patients were in complete heart block. Fuller clinical details are being published elsewhere (Harris, Bluestone, Busby, Davies, Leatham, and Siddons, in press).

Throughout the period under consideration (up to 30 September 1964) 143 pacemakers were implanted in these 79 patients. The first two were of Swedish make*, but subsequently pacemakers designed by one of us (J.G.D.) have been used exclusively, some of them having been made in the electronics department of St. George's Hospital and some to our design + . In treating 19 of our early patients a number of prototype units were tried, some from outside with only a receiver unit implanted, and others containing their own battery for power; some could be turned on and off, speeded up, and slowed down from outside. However, as a result of experimental work done in this hospital (Sowton, 1963), an early decision was taken to concentrate on the development of a fixed-rate pacemaker powered by miniature mercury cells within the implanted unit. There was no time for prolonged laboratory or animal trials since patients with complete heart block in whom Stokes-Adams attacks threatened life required pacing without delay. Thus it has proved necessary to modify the apparatus as faults revealed themselves during clinical use (see Table I).

The earliest patients were treated with a pacemaker implanted in the rectus sheath with wires leading to the surface of the heart (epicardial system) (Fig. 1). A high proportion developed sinuses and many of the pacemakers had to be taken out. Before this complication reached serious propor-

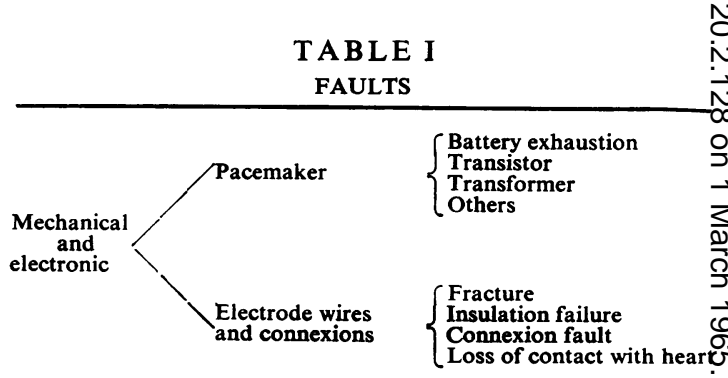

Tissue reaction $\left\langle\begin{array}{l}\text { Sinus and sepsis development } \\ \text { Rise of threshold required for stimulation }\end{array}\right.$

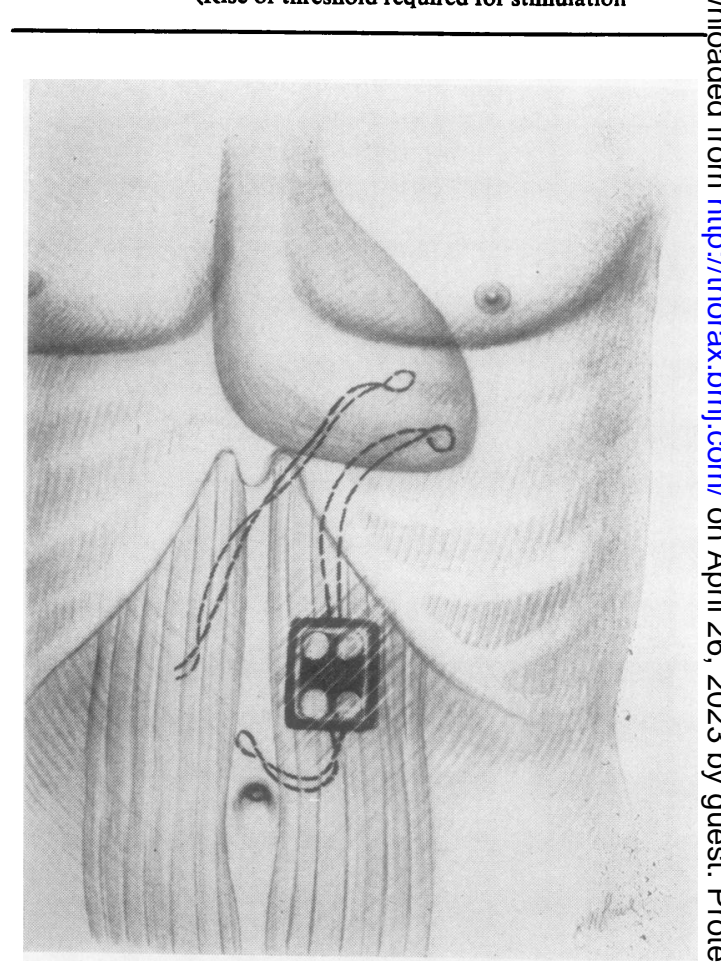

FIG. 1. Epicardial electrode with abdominal pacemaker and spare epicardial wire-electrode. 


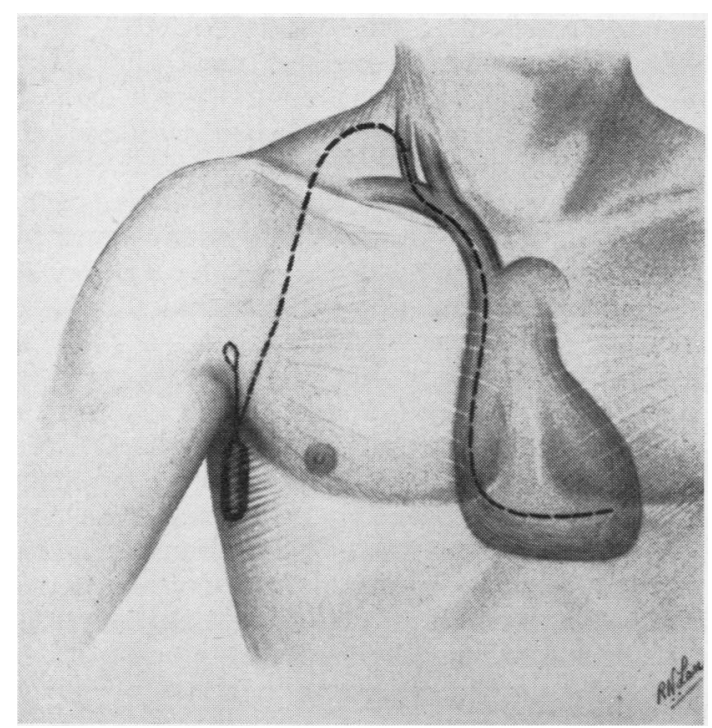

FIG. 2. Endocardial electrode with axillary pacemaker.

tions, we had been developing an alternative method which avoids a thoracotomy, using an electrode on the end of an insulated wire passed via the jugular vein to make contact within the right ventricle (endocardial system) (Fig. 2). Our current technique uses such an electrode joined to a pacemaker implanted in the axilla.

\section{PACEMAKER DESIGN}

In the original design of the pacemaker in 1961 a number of conditions were arbitrarily chosen on the evidence available, the principal one being that with a unipolar stimulating system a pulse of 5 volts at 2 milliseconds would always be above the threshold required. The stimulator (Davies, 1962) was of constant voltage type so that pacing would not cease with failure of the insulating material on the wires leading to the heart.

Concurrently with the implantation of the first of these units at St. George's Hospital, the first endocardial wire electrode system was used according to the technique adopted at the Montefiore Hospital, New York (Furman and Schwedel, 1959). (The insulated wire with bare platinum tip for contact with the endocardium of the right ventricle is often erroneously referred to as an electrodecatheter; it has no lumen.) The endocardial system with an external pacemaker was advantageous in that it avoided a thoracotomy but involved the risk of septicaemia from organisms tracking from the outside along the wire into the blood stream. As experience increased and no complications of myocardial perforation or from intravascular clotting presented, it was a logical development to use the endocardial wire electrode in association with an implanted pacemaker. The design of the first four units made for this system proved unsatisfactory as the power required was underestimated; three of the units had to be removed within 20 days. This experience led to the production of the pacemaker currently used in conjunction with an endocardial electrode (Mark VI).

In 1963 there were signs that the units (Mark II) used with epicardial electrodes would not last the predicted three to five years, and they were replaced when necessary. This provided the opportunity to measure threshold values recorded below and showed that the original design factors gave an unnecessarily large margin of power. The early units putting out a stimulus of 5 volts for 2 milliseconds had a power level of about 200 microjoules, whereas the average requirement was only 25 microjoules. The model was therefore modified by shortening the pulse duration to lower the power level and hence increase its life. Some indication of the final power threshold may be obtained from initial readings allowing for the fact that a plateau of up to 10 times the original is reached after two to three weeks.

Experience has shown that after the initial rise the mean level required for endocardial, as opposed to epicardial, stimulation is only 4 microjoules, so a unit delivering 15 to 20 microjoules has been developed for such endocardial pacing (Mark VI). These two types, Mark II and Mark VI designed for epicardial and endocardial pacing respectively, are basically similar, and, with slight changes during construction, an endocardial model can be made to produce a longer impulse and therefore sufficient power for epicardial stimulation.

Also in 1963 a fault developed in a large batch of transistors, and the circuit was modified to exclude the use of this transistor and of one other component. This alteration was made at the same time as the shortening of the pulse duration and did not involve the addition of any extra components. There have been no other transistor failures. In this year also there were two transformer failures, and the newer models have a differently constructed transformer which should eliminate this particular fault.

The original circuit was so designed that when battery exhaustion was impending, the rate speeded up to warn that replacement was required. In some patients an inadequate watch was kept, and the rate increased to a dangerous level before the patient returned to hospital for unit replacement. In the newer models the circuit has therefore been arranged 
so that battery exhaustion is heralded by slight slowing rather than speeding, which avoids the dangers of too rapid stimulation. Impending battery exhaustion is also checked at routine visits by the patients to the hospital clinic by measuring the pacemaker impulse on the E.C.G. with an oscilloscope.

Experience showed that the original units, if set at a rate of 65 per minute, would function for 65 to 80 weeks before battery exhaustion. $W$ ith the reduced power output of the present models it is calculated that the life of these units should be four years. Recently, owing to endocardial electrode fracture, an axillary unit (Mark Vl) has been removed after four months' use, and laboratory tests suggest that 7 per cent. of the battery power has been used, substantiating the hope that these units will last this predicted period.

Some failures of pacemakers have not been completely explained. For example, a failure which appeared to be that of a defective component, such as a resistor, may have been the consequence of mechanical stresses in the potting medium, only becoming evident after a period of months. Care is needed in interpreting component failure, which may basically be caused by the conditions which pertain for an implanted pacemaker. It is inevitably subjected to certain mechanical strains. Battery leakage or the ingress of body fluids could affect electronic parts, and this process might be accelerated by the body temperature. Some evidence is available to support this theory from experience using pacemakers of identical design placed externally, where failure of components has been appreciably lower.

\section{WIRES AND ELECTRODES}

Several types of electrodes and wires for epicardial pacing were tried until an electrode system was evolved using a triple spiral wire, part of which was free of insulation and acted as an electrode (Siddons, 1963). This system has as far as fractures are concerned been remarkably successful, without a single wire failure or insulation leak. Stainless steel was used at first for both epicardial (negative) and indifferent (positive) electrode wires, but in some cases in which sepsis developed, the indifferent electrode tended to fragment, and platinum is now used in place of stainless steel for this electrode. Stainless steel is still preferred for the epicardial electrode owing to its more suitable mechanical properties. There has never been fragmentation on the epicardial electrode, and, since changing to platinum, this has not occurred with the indifferent electrode.
For endocardial wire electrodes, C50 No. $\frac{5}{\circ}$ Electrode Catheters* have been used. Though per- $\frac{C}{D}$ foration of the heart can occur if such catheters $\overline{\bar{s}}$ are passed from an arm vein, we have not seen this $\widetilde{\Phi}$ complication using a neck vein, provided a No. 5 을 is used and not a stiffer one. In two cases, failurew to pace was traced to cracking insulation at a site of $\overrightarrow{0}$ acute angulation in the neck. In both cases the electrodes had been in situ for four months.

THE UNIPOLAR SYSTEM For epicardial pacing a uni- $-\vec{x}$ polar system has been adopted rather than the bi- $\stackrel{-}{\circ}$ polar, with both electrodes on the heart, as has beeniv used by several A.merican workers. It is claimed that $\vec{N}$ the threshold is 10 per cent. lower with the bipolar ${ }^{\infty}$ system (Chardack, 1964). This advantage is out-? weighed by other considerations. Fragmentation of $\vec{z}$ electrodes, mentioned previously, has only been observed at the anode and appears to be avoided by using platinum for this electrode and wire. However, $\overrightarrow{\mathscr{A}}$ if it is placed on the heart, the wire must withstand 8 the constant cardiac movement, and stainless steel ${ }^{\sigma}$ has more desirable mechanical properties for this purpose. Thus it is logical to use stainless steel for the cathode on the heart but platinum for the anode⿳亠二口犬 placed at a site where there is minimal movement. If a pacemaker with a greater output than our@ design is used with a unipolar arrangement, twitch $-\vec{\overrightarrow{ }}$ ing of skeletal muscle can prove troublesome. This 3 is avoided with pacemakers of our design provided care is taken in siting the indifferent electrode away from muscle and nerve.

There are further considerations with endocardialo pacing where the reliability of the catheter electrode ${ }_{x}$ becomes paramount. We have been reasonablyo satisfied with the unipolar C50 electrode-catheters. referred to above, but with six of the eight bipolaro electrode-catheters (C51 and C52) that we have used $₹$ fractures have occurred. These were used with음 external pacemakers and thus do not feature in this series.

\section{CONNEXION FAULTS}

Designing a suitable means of connecting the $\tilde{O}$ insulated electrode wires to the pacemaker proved N difficult. It must be possible to remove a pacemakero and connect a replacement under sterile operativeo conditions. The joint must not only make good electrical contact but the insulation must be water-? tight. Seepage of tissue fluid into the pacemaker 0 unit may interfere with its correct functioning. The simple mechanical method with two nylon screws holding the bare wire and its insulation and the use $\mathbb{Q}_{\overparen{Q}}^{\mathbb{P}}$ of silicone grease rammed into any crevices by the 
screw has proved reasonably satisfactory, though the technique required at operation is tedious for the surgeon unaccustomed to the small screwdriver. Two faults occurred during the period of development of the method. Subsequent to the development of the present technique, in only two patients was good contact not achieved at operation so that shortly afterwards pacing was intermittent. On both occasions readjustment of the screw cured the fault, and in the future, with greater care over assembly, it is hoped that this defect will not reappear.

\section{LOSS OF CONTACT WITH THE HEART}

When sepsis develops around the epicardial electrode the mechanical fixation to the heart may give way. This has not occurred in the absence of sepsis.

Provided the platinum tip of an endocardial electrode has remained in contact with the wall of the ventricle, pacing can continue. There have been reports of the bare platinum of an endocardial electrode becoming covered with thrombus which may interfere with pacing (Parsonnet, Zucker, Gilbert, and Meyers, 1964). This has not been observed in our cases perhaps because we always use the endocardial electrode as the cathode.

In six cases it has proved necessary to reposition the electrode as it has become displaced, but this has almost always occurred within two days of placing the electrode, and one can thus be confident that displacement is extremely unlikely by the time the patient is well enough to leave hospital.

In three patients pacing became intermittent and no satisfactory explanation was found. In two of them pacing has continued satisfactorily after the substitution of a new electrode wire (though no fault could be found in the discarded wire). The third is now being paced with an endocardial electrode attached to an external pacemaker, the cause of failure offering a complex technical problem as yet unsolved.

\section{WOUND SINUSES}

The most serious complication was the development of a sinus in the thoracotomy scar or over the abdominal pacemaker. This occurred in 23 of the 55 patients* in whom abdominal pacemakers were implanted, often necessitating their removal. A few wounds did not heal from the start, but in most cases healing was satisfactory, sometimes with no unusual amount of post-operative pain. Pain developed some months later and was followed

\footnotetext{
* Four patients who had their units implanted in other hospitals, but were subsequently treated at St. George's, are excluded from these numbers but included in Table III. Fig. 4 shows that in 21 the pacemakers were removed for this reason
}

by a sinus (longest, nine months). Occasionally it was possible to aspirate fluid from beneath the scar before the sinus appeared, and no organisms were grown from such fluid, whether or not antibiotics had recently been given. Smears from the freshly developed sinus proved sterile or grew Staphylococcus albus. It was only when a sinus had been established for many weeks that pathogens such as Staphylococcus pyogenes became established. At some stage, usually weeks or months after the sinus had appeared, suppuration was severe enough to require exteriorization of the pacemaker. After this step the threshold required for stimulation still rose, making it necessary to pace by an alternative means. As a first step the spare myocardial wire which had been placed at the original thoracotomy was brought out and, provided its threshold was suitably low, it was used. In many cases, however, this threshold was also high and it was necessary to change to pacing with an endocardial system. In nine patients suppuration was severe enough to necessitate removal of the wires and electrodes, for healing never occurs with both sepsis and foreign bodies. In two patients, abscesses around the electrode on the ventricular wall resulted in severe haemorrhage at operative removal.

The aetiology of these sinuses has not been finally proved. In three patients the sinus developed early, and it must be assumed that sepsis was the cause either from incomplete sterilization of the apparatus or from contamination at operation. In the remainder the sinus did not develop for an average period of 17 weeks. This delay in sinus formation, the failure to obtain pathogenic organisms until weeks after sinuses had developed, and the fact that sinuses developed with apparatus sterilized by various techniques suggest that sepsis is unlikely to have been the cause. The pacemaker itself will not stand autoclaving or gamma irradiation and was sterilized in chlorhexidine solution or in formalin gas, using an elaborate evacuation technique. The wires and electrodes were sterilized by these methods or by autoclaving, and since tissue fluid can seep up some of the wires, tests were carried out to establish that by this means the lumen was effectively sterilized. Sinuses developed in a significant proportion of cases with each method of sterilization. It is thought that organisms usually considered nonpathogenic, such as Staph. albus, might, in association with a foreign body, survive and cause sinus formation, but failure to obtain this organism from cultures of aspirated fluid or from a freshly formed sinus in eight cases is evidence against this explanation.

If the sinus development was not due to sepsis 
it must be attributed to a reaction to the surface material of the apparatus. The silicone rubber (Dow Corning No. 502) covering the wires has been implanted in human beings frequently both as cover for pacemakers of other design and for other purposes and has proved remarkably inert. Sinus formation is unlikely to be due to the passage of the electric current, as similar stimulation has been used extensively by others without sinus formation. Following the example of others (Elmqvist and Senning, 1960) the pacemaker itself was potted in an epoxy resin (MY 753 Ciba Ltd.). Others who have used Elmqvist's Elema pacemaker have not reported sinus formation (Landegren and Biörk, 1963), but since the material is made up in batches using a hardener, it is not possible to achieve an identical article in every batch, thus allowing the possibility of the end product being irritant in some instances. Recently therefore the epoxy resin has been covered with silicone rubber, a softer material and one for which there is plenty of evidence of inertness in the tissues, but it is too early to be confident that this has solved the problem.

Consideration must be given to the possibility that the chemical sterilizing agents which are partly absorbed into the epoxy resin or silicone rubber are given off after implantation and cause irritation resulting in sinuses. The hardener used in the preparation of the epoxy resin might act in a similar manner since some units were implanted only a short time after the completion of manufacture.

Finally, the site of implantation may be of importance, and our practice has differed from that of American workers who have implanted their units subcutaneously rather than in the rectus sheath. In small and especially in thin patients there may be mechanical difficulty in obtaining good skin closure at any site.

in short, there has been a high incidence of sinus development in our practice using epicardial wires. No other workers have reported this complication in more than a very occasional case although the same materials and sterilizing agents are widely used. It is hoped that the trouble has been eliminated by covering the units with silicone rubber and by leaving them for some days after sterilization before implanting them, and as it is possible that the rectus sheath is an unsatisfactory site for pacemaker implantation, we propose in future to place them subcutaneously, but in most cases now we use an endocardial-axillary system to avoid a thoracotomy.

In contrast to the high incidence ( 23 of 55 ) of sinus development with the epicardial technique with the pacemaker in the rectus sheath, there has been much less wound trouble with the endocardial technique implanting the pacemaker in the axilla ( 3 in 37, see below), but these figures must be interpreted with caution as experience with the endocardial system has not been so prolonged. Moreover, $\overparen{\mathbb{D}}$ most of the abdominal units were covered with $\frac{0}{2}$ epoxy resin and most of the axillary units with कै silicone rubber; and the two groups are not entirely $\overrightarrow{0}$ comparable in some other respects. Total experience $\overrightarrow{-}$ with the epicardial-abdominal system is in $59 \vec{\omega}$ patients over four and a quarter years compared with the endocardial-axillary system in 37 patients over 11 months ( 17 had periods with both systems). 을 The mean period of pacing in the 37 endocardial- iv axillary patients was six months.

The three patients with axillary implants who developed local trouble did not run courses at all similar to the abdominal patients. In one, the $\vec{z}$ unit was placed too close to the skin suture line and protruded shortly after the stitches had been taken $\stackrel{\widehat{\rho}}{\supset}$ out. In another, the wound gaped on removal of the $\vec{\theta}$ stitches, resulting in some sepsis which was con- of trolled by antibiotics; after resiting the unit the wound healed satisfactorily. The third patient was a complex problem associated with septicaemia, s which may have resulted from previous temporary pacing with an endocardial venous electrode but an external pacemaker. The operation of implantation $\stackrel{\mathbb{Q}}{2}$ had been carried out as an emergency without full $\overrightarrow{\hat{O}}$ sterile precautions. In none has there been the 3 sequence of pain with late sinus development, as occurred so often with the epicardial electrodes? and the pacemaker in the rectus sheath.

\section{THRESHOLD}

Measurements of the thresholds necessary for stimulation have been recorded after varying 0 periods of pacing whenever the opportunity pre- $₹$ sented. $\mathrm{W}$ ith epicardial stimulation this amounts to 을 1.3 volts at 1 millisecond and 2.5 microjoules rising $>$ to 2.5 to 3.0 volts and 25 microjoules after about 14 을. days, where it remains static. In contrast to epicar- $N$ dial stimulation, with endocardial electrodes the equivalent figures are 0.5 volt at 1 millisecond with $\tilde{\text { S }}$ a power level at about 0.5 microjoule rising to 2.5 to 3.0 volts and 4.0 microjoules when the stable level is reached.

However, there have been exceptions with epi- $\frac{\stackrel{\circ}{\mathbb{D}}}{\mathbb{D}}$ cardial stimulation, and in four cases a plateau was $\stackrel{\oplus}{?}$ reached only after six to eight weeks at 6.0 volts and 0 about 140 microjoules. In these cases there was no evidence of sepsis, and it was concluded that an unusual amount of fibrosis around the myocardial $\stackrel{\mathbb{\alpha}}{\mathscr{\alpha}}$ electrode was the main cause for these abnormally high threshold readings. In one of these cases 
FIG. 3. Indicates the length of time each individual has been paced to 30 September 1964 or the time of death. (In three patients pacing was omitted on clinical grounds.) with implanted pacemaker; with exterior pacemaker.
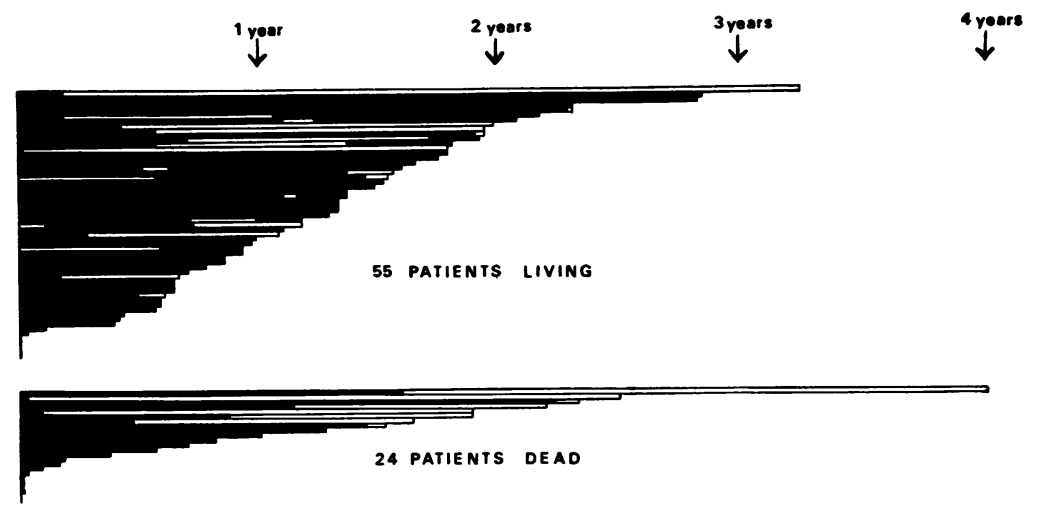

there was an excessive number of stitches around the point of contact which probably accounted for the reaction. It must be emphasized that tissue damage around the myocardial electrode may lead to conditions necessitating a much higher level of power than would normally be necessary.

In the presence of sepsis, threshold measurements vary considerably even from day to day. In one case it rose to 9 volts at 2 milliseconds and 310 microjoules. (This was by an external pacemaker along wires which had been exteriorized.) In a few patients with bipolar stimulation and in the presence of sepsis, a rising threshold could be halted and even reversed temporarily by interchanging the positive and negative connexions to the pacemaker. This at times caused a dramatic lowering of the voltage required from 6 to $2 \cdot 5$. At present there is no satisfactory explanation of this mechanism. Attempts to correlate changes in threshold with a change in electrical impedance between the electrodes has revealed no significant association between the two parameters, nor has the impedance varied appreciably with the passage of time, fibrosis or sepsis.

Figure 3 shows graphically the periods of pacing the 79 patients. Figure 4 shows the duration of implantation for the 121 (Mark II and Mark VI) pacemakers indicating the reason for removal in those not still implanted. Table JI lists the major faults and the steps taken to eliminate them. A discussion on methoas of investigation of patients when faults develop has been published elsewhere (Sowton and Davies, 1964).

Of these 79 patients, 55 were still surviving on 30 September 1964 . Some have only been paced for a brief period, the longest over three years. The mean period of pacing in the survivors is $11 \frac{1}{2}$ months. Seven of the 24 deaths occurred within a month of starting pacing (four apparently in ventricular

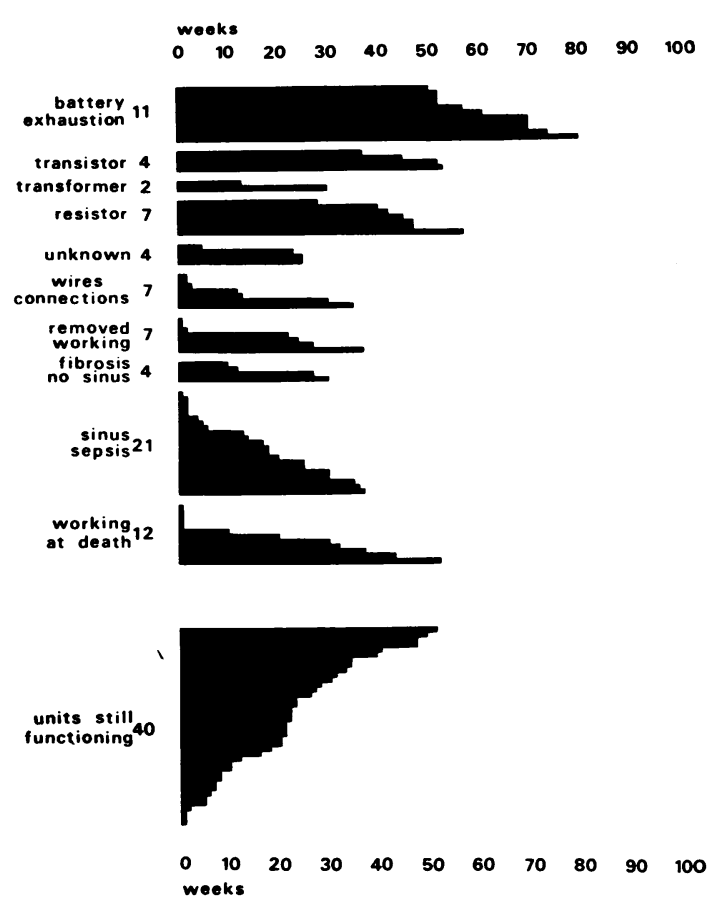

FIG. 4. Each column represents an implanted pacemaker and shows in weeks its functioning life. As mentioned in the text, the seven failures apparently due to 'resistors' may have other causes. Those pacemakers 'removed working' were taken out for various clinical reasons. 'Fibrosis no sinus' covers four patients in whom the threshold required for stimulation rose, and in some this was due to fibrosis around the epicardial electrode. The pacemaker itself was functioning satisfactorily on removal in this group and in the group labelled 'sinus/sepsis', which is fully discussed in the text. The last group of columns represents those pacemakers still functioning on 30 September, 1964. 
TABLE II

\begin{tabular}{c|c}
\hline Transistor failures (4) & $\begin{array}{l}\text { Transistor eliminated : } \\
\text { no failures in 99 units } \\
\text { Modified transformer: } \\
\text { no failures in 95 units } \\
\text { With power output reduced } \\
\text { four years' life anticipated }\end{array}$ \\
$\begin{array}{c}\text { Transformer failures (2) } \\
\begin{array}{c}\text { With early units battery } \\
\text { exhaustion at about one year } \\
\text { (11 cases) }\end{array}\end{array}$ & $\begin{array}{l}\text { No failures or displacement } \\
\text { from heart with current tech- } \\
\text { nique in 108 implants }\end{array}$ \\
\hline \begin{tabular}{c} 
Epicardial \\
and \\
Electrode \\
wires \} \begin{array} { c } { \text { Endifferent } } \\
{ \text { 'catheterdial } } \\
{ \text { two fractures } } \end{array} & New designs under trial \\
\hline
\end{tabular}
\end{tabular}

fibrillation, which could have been the result of pacing). Some of the other deaths may be attributable in part to the complications of pacing, but only in one has pacemaker failure directly resulted in death. Four of the deceased patients had been successfully paced for over two years and the longest for over four years. It is to be hoped that by modifying the apparatus as defects become apparent and by great attention to detail, both in preparation of the units and in their implantation, our results will improve. The survival so far of all the 33 patients paced by our current technique (endocardial-axillary with Mark II or VI pacemaker) seems to justify our continued efforts, though these patients have as yet only been paced for a mean period of six months.

\section{SUMMARY}

Table II shows that the original pacemaker design has been modified with growing experience. It is reasonable to hope that with the present techniques the apparatus should pace satisfactorily for several years, requiring replacement by a minor operation perhaps every thi ee to five years.

The initial technique with epicardial electrodes and a pacemaker buried in the abdominal wall was associated with a high incidence of sinus formation followed by sepsis. The cause of this complication is not clear, but it appears to have been eliminated in the current technique with endocardial electrodes and a pacemaker buried in the axilla which also has the advantage of avoiding a thoracotomy. The last 33 patients treated by this method survive.

For the last year the British Heart Foundation have been making a grant to the Cardiac Department of St. George's Hospital in support of this work.

\section{ADDENDUM}

In the last three months there has been one further death, in a patient who had been paced for two and a half years. The electrode wire in the neck ulcerated through to the surface, allowing the risk of sepsis $i$ tracking along the wire as when an external pacemaker is used. Death was due to renal failure following septicaemia.

Two more patients with abdomino-epicardial systems have had their pacemakers exteriorized following sinus formation.

The endocardial electrode has given trouble in eight cases, the electrode tip losing electrical contact with the ventricular wall in four, the electrode wire fracturing in two, and the insulation failing in two others. This emphasizes the necessity of improving the design of the endocardial electrode if this system is to continue to be the method of choice.

In the last 11 months 40 pacemakers (Mark VI) have been made and implanted in this hospital. To date, no faults have developed in these units.

\section{REFERENCES}

Chardack, W. M. (1964). Heart block treated with an implantable pacemaker. Progr. cardiovasc. Dis., 6, 507.

Davies, J. G. (1962). Artificial cardiac pacemakers for the long-term treatment of heart block. J. Brit. Instn Radio Engrs, 24, 453.

Elmqvist, R., and Senning, A. (1960). An implantable pacemaker for the heart. In Medical Electronics. Proc. 2nd int. Conf. Paris, June 1959. Ed. C. N. Smyth, p. 253.

Furman S and Schwedel, J. B. (1959). An intracardiac pacemake for Stokes-Adams seizures. New Engl. J. Med., 261, 943.

Harris, A. M., Bluestone, R., Busby, Eileen, Davies, J. G., Leatham, A., and Siddons, A. H. M. The management of heart block. In press.

Landegren, J., and Biörk, G. (1963). The clinical assessment and treatment of complete heart block and Stokes-Adams attacks. Medicine, 42, 171 .

Parsonnet, V.. Zucker, I. R., Gilbert, L., and Meyers, G. H. (1964). A review of intracardiac pacing with specific reference to the use of the dipolar electrode. Progr. cardiovasc. Dis., 6, 472.

Siddons, A. H. M. (1963). Long-term arificial cardiac pacing ; experience in adults with heart block. Ann, roy. Coll. Surg. Engl., 32, 22.

and Davies, J. G. (1963). A new technique for internal cardiac pacing. Lancet, 2, 1204.

Sowton, E. (1963). Unnublished M.D. thesis.

- and Davies, J. G. (1964). Investigation of failure of artificial pacing. Brit. med. J., 1,1470 . 